\title{
Child abuse and aggressivity: the childhood of serial killer, spree killer and mass murderer
}

\section{Maltrattamento infantile e aggressività: l'infanzia di serial killer, spree killer e mass murderer}

\section{Abuso infantil y agresividad: la infancia de serial killer, spree killer y mass murderer}

\author{
Giulia Pitzanti
}

Dottoressa Magistrale in Investigazione, Criminalità e Sicurezza Internazionale, Università degli Studi Internazionali di Roma (UNINT), Italy

\begin{abstract}
This article aims at analysing the phenomenon of child abuse, the consequences that it may produce in the different evolutionary stages and the role it plays in the manifestation of aggressive behaviour in adulthood. Also, it examines the relationship between the different types of abuse suffered during childhood and the development of criminal phenomenologies such as serial killers, spree killers and mass murderers.
\end{abstract}

\section{RIASSUNTO}

Il presente articolo mira all'analisi del fenomeno del maltrattamento infantile, delle conseguenze che questo può produrre nei differenti stadi evolutivi e del ruolo che ricopre nella manifestazione di comportamenti aggressivi in età adulta. Inoltre, viene esaminata la relazione che intercorre tra le diverse tipologie di abuso subito durante l'infanzia e lo sviluppo di fenomenologie criminali quali serial killer, spree killer e mass murderer.

\section{RESUMEN}

El presente artículo tiene por objeto analizar el fenómeno del abuso infantil, las consecuencias que éste puede producir en las diferentes fases evolutivas y la función que desempeña en la manifestación de comportamientos agresivos en la edad adulta. Ademàs, se examina la relación que existe entre los diferentes tipos de abuso sufrido durante la infancia y el desarrollo de fenomenologías delictivas como serial killer.

\section{Il maltrattamento infantile}

Dal confronto tra le ricerche riguardanti le segnalazioni di abuso e le ricerche retrospettive, emerge che i fenomeni di violenza sui bambini sono ampiamente più diffusi rispetto a quanto mostrano le stime ufficiali, in particolar modo in Italia, in quanto non è presente un registro nazionale.

Secondo il parere di Di Blasio, P. e Rossi, G. (2004), una definizione completa è stata proposta dal "Consultation on Child Abuse and Prevention" del WHO (2002), che sottolinea come "per abuso all'infanzia e maltrattamento debbano intendersi tutte le forme di cattiva salute fisica elo emozionale, abuso sessuale, trascuratezza, negligenza, sfruttamento commerciale o altro che comportano un pregiudizio reale o potenziale per la salute del bambino, per la sua sopravvivenza, per il suo sviluppo o per la sua dignità nell'ambito di una relazione caratterizzata da responsabilità, fiducia o potere". Da questa definizione inizia ad emergere la varietà del fenomeno: l'abuso non è esclusivamente commissivo, può manifestarsi anche mediante omissione (negligenza, trascuratezza); inoltre, non precludendo l'abuso al solo ambiente familiare, la definizione permette di includere maltrattamenti e violenze che si manifestano anche in contesti extrafamiliari.

Il maltrattamento non può essere considerato come un quadro globale e indifferenziato messo a confronto con una condizione di non maltrattamento altrettanto generica. È invece necessario specificare in maniera sempre più precisa e articolata le numerose dimensioni implicate nel fenomeno. Una delle dimensioni maggiormente analizzate risulta essere la tipologia. Nonostante la suddivisione in tipologie sia necessaria dal punto di vista descrittivo, questa non è rappresentativa della complessità del fenomeno e non è, inoltre, possibile eseguire una netta distinzione tra i differenti tipi di maltrattamento, in quanto essi si dimostrano essere prevalentemente compresenti (Emiliani e Simonelli, 1997).

Verrà di seguito riportata la classificazione proposta da Di Blasio (2000), basata su quella stilata dal Child Protection Register inglese (Gibbons, Conroy e Bell, 1995), che identifica quattro sub-tipologie del maltrattamento, riprese poi nelle Linee Guida delineate da Save The Children Italia (2018): la trascuratezza, il maltrattamento fisico, l'abuso sessuale e l'abuso emozionale.

Il termine trascuratezza, o patologia delle cure, identifica una grave e costante negligenza nei confronti del bambino; si verifica nel momento in cui i genitori non sono in grado di proteggerlo 
dai vari pericoli a cui può essere esposto, inclusi freddo e fame, oppure nel caso in cui essi falliscano in un determinato ambito di allevamento, minando la salute e lo sviluppo del bambino (Di Blasio, 2000). All'interno di questa categoria sono incluse:

- l'incuria, che indica la carenza/assenza di cure;

- la discuria, ovvero la presenza di cure che risultano non essere in linea con la fase evolutiva e le necessità del bambino;

- l'ipercuria, ossia la somministrazione eccessiva di cure.

L'ipercuria comprende la Sindrome di Munchausen per Procura e il Medical Shopping per Procura. Il primo indica un disturbo psicopatologico che porta i soggetti che ne sono affetti a spostare la convinzione di malattia sui figli: i genitori (nella quasi totalità dei casi la madre) immaginano sintomi e malattie del figlio, proiettando le proprie insoddisfazioni e problematiche su esso, che di conseguenza viene sottoposto a cure inopportune e accertamenti clinici non realmente necessari (Karlin, 1995). Il secondo è un disturbo nevrotico-ipocondriaco che si presenta in casi di genitori ansiosi ed eccessivamente preoccupati per la salute del proprio figlio, tanto da rivolgersi a numerosi specialisti per avere rassicurazioni anche per lievi patologie percepite immotivatamente come grande minaccia alla vita del bambino (A.P.A., 2013).

In quest'ambito si inserisce, inoltre, la trascuratezza istituzionale, che si concretizza a partire da soggetti interni a istituzioni/organizzazioni ai quali i minori sono stati affidati per motivazioni quali cura, custodia e educazione. Questa riguarda l'assenza/carenza di cure adeguate da parte dei servizi istituzionalmente preposti alla tutela dei minori, la sottovalutazione di segnalazioni esplicite $\mathrm{o}$ implicite che possano orientare attività protettive e preventive, l'assenza del rispetto della privacy del minore e l'eccessiva burocratizzazione (Novello, 2018).

La trascuratezza si manifesta attraverso la scarsa cura nella pulizia del bambino, la denutrizione, l'assistenza medico-sanitaria assente o carente, l'abbigliamento inadeguato all'età o alla stagione, l'esclusione dall'obbligo scolastico o la distorsione delle abitudini alimentari. I minori trascurati possono apparire stanchi, inibiti e tristi o, al contrario, iperattivi e affetti da disturbi d'attenzione, potrebbe essere evidente l'assunzione del ruolo genitoriale da parte del figlio nei confronti dei genitori (Di Blasio, 2000).

L'abuso fisico implica un danno físico procurato dai genitori, o il loro fallimento nel prevenirlo. In base alla gravità delle lesioni subite dal bambino è possibile classificare gli abusi fisici in:

- abuso di grado lieve: le lesioni non necessitano alcun ricovero;

- abuso di gravo moderato: le lesioni causano la necessità di ricovero, come nel caso di ustioni, traumi cranici, fratture, etc.; - abuso di grado severo: il bambino viene ricoverato in rianimazione e subisce gravi conseguenze neurologiche fino a giungere alla morte (Di Blasio, 2000).

I segni fisici dell'abuso fisico sono generalmente bruciature da sigarette o ustioni, contusioni, segni di soffocamento, ecchimosi, morsi, traumi cranici, fratture multiple, segni di avvelenamento etc. Proprio i segni tangibili e la necessità di ricorrere alle cure ed all'intervento medico rendono l'abuso fisico uno dei sottotipi del maltrattamento maggiormente studiato (Emiliani e Simonelli, 1997).

I minori vittime di abuso fisico possono presentare reattività esagerata, scoppi improvvisi d'ira, rifiuto del contatto fisico o ricerca di questo con modalità distorte, atteggiamento timoroso nei contesti estranei e arrogante nei contesti d'origine (Di Blasio, 2000).

L'abuso sessuale riguarda qualsiasi attività sessuale che intercorre tra un adulto e un/una bambino/a che, per ragioni di immaturità psicologica e/o affettiva, o per condizioni di dipendenza dagli adulti, non è ritenuto in grado di poter compiere scelte realmente comprese o di avere adeguata consapevolezza del significato e del valore delle attività sessuali in cui viene coinvolto (Save the Children Italia, 2018).
Rientrano nella categoria di abuso sessuale i rapporti sessuali veri e propri, toccamenti e/o rapporti orali tra adulti e bambini, sfruttamento sessuale, violenza sessuale assistita, avvio alla prostituzione infantile, incoraggiamento/costrizione di bambini alla vista di atti sessuali, induzione alla visione di materiale pornografico o utilizzo dei bambini per la sua produzione (Save the Children Italia, 2018).

I segni fisici e comportamentali ${ }^{1}$ riscontrabili potrebbero essere contusioni, graffi, difficoltà nella deambulazione o nel sedersi, infezioni alle vie urinarie, umore negativo duraturo, comportamenti sessuali inadeguati all'età, autolesionismi (Di Blasio, 2000).

L'abuso emozionale, o maltrattamento psicologico, si riferisce a una relazione emotiva inappropriata e dannosa caratterizzata da pressioni psicologiche, ricatti affettivi, indifferenza verso i bisogni psicologici, educativi e sociali del bambino, rifiuto, isolamento forzato, violenza assistita, denigrazione e svalutazioni che danneggiano o inibiscono lo sviluppo di competenze cognitivo-emotive fondamentali (Di Blasio, 2000).

All'interno della presente categoria viene inserito il fenomeno dell'Alienazione Parentale (Parental Alienation - PA): i bambini, che vengono "programmati" dal genitore "alienante", mettono in atto una campagna denigratoria nei confronti del genitore "alienato", e gli scenari denigratori propri dei bambini integrano e completano quelli provenienti dal genitore "programmante", che impiega comportamenti alienanti (Alienating Behaviors - AB) (Baker e Verrocchio, 2016).

Ulteriore tipologia di maltrattamento riconducibile alla presente categoria è la violenza assistita intrafamiliare, con cui si fa riferimento ad atti di violenza fisica, verbale, psicologica, sessuale ed economica, ottemperati nei confronti delle figure di riferimento o affettivamente significative, dei quali il bambino può avere esperienza diretta (nel caso in cui avvenga all'interno del suo campo percettivo) o indiretta (nel caso in cui il bambino sia a conoscenza della violenza o ne percepisca gli effetti). Questa tipologia comprende anche i maltrattamenti compiuti da minori, o eseguiti dai familiari verso gli animali (Luberti e Pedrocco Biancardi, 2005; Novello, 2018).

L'abuso emotivo si esprime attraverso critiche, ironia, sarcasmo, disprezzo, modalità verbali fortemente svalutanti e sadiche, e potrebbe portare a segni comportamentali dei bambini quali atteggiamento timoroso o aggressivo, continua svalutazione del sé, ansia nelle separazioni o comportamenti di sfida. A livello emotivo i bambini potrebbero presentare scarsa autostima, assenza di fiducia di base, tristezza, inibizione e assenza di slancio vitale (Di Blasio, 2000).

\section{Le conseguenze psicologiche del maltrattamento infantile}

Il maltrattamento, in ogni sua sfumatura, presenta sin dal periodo neonatale un insito rischio di alterazioni psicologiche che si protraggono poi nell'età adulta.

\footnotetext{
1 Gran parte degli abusi sessuali sui minori comporta toccamenti, carezze, e altre attività che non lasciano segni, inoltre, l'attesa che precede la denuncia da parte dei bambini permette a eventuali lesioni presenti di risanare. Per questi motivi le dichiarazioni del bambino costituiscono indizi fondamentali delle molestie e, nonostante la rilevanza di esami medici in caso di sospetto abuso, l'accertamento deve basarsi su una valutazione multi-sistemica (clinico, psicologico-giuridico e psico-sociale).
} 
Beeghly e Cicchetti (1994) hanno eseguito uno studio svolto su un campione di bambini maltrattati di età compresa fra i 31 ed i 33 mesi, dimostrando che le conseguenze del maltrattamento si manifestano fin dal periodo neonatale attraverso un disturbo della sfera emozionale. Questo si mostra alquanto precocemente sotto forma di una disfunzione in due ambiti distinti: il neonato sembra comprendere in modo errato e distorto le reazioni emotive espresse dalle altre persone, con una sorta di misconoscimento di queste, al quale segue la messa in atto di reazioni notevolmente discrepanti con le richieste altrui.

In particolare, già a partire dai tre mesi di vita, i neonati maltrattati sono in grado di differenziare in modo significativo la paura tra le emozioni e manifestano precocemente reazioni di paura in risposta al dolore (palesando un disturbo del sistema regolatorio del sé), mentre i neonati non maltrattati rispondono all'esperienza del dolore primariamente con la rabbia e la protesta.

Le difficoltà di comprensione ed espressione delle emozioni vengono accompagnate anche da una ridotta capacità di utilizzare un linguaggio riferito agli stati interni, in quanto il clima disfunzionale relazionale presente nella famiglia maltrattante non favorisce l'espressione linguistica delle emozioni, bensì rafforza stili interattivi e relazionali evitanti (Coster et al., 1989; Carlson et al., 1989). I bambini del campione sembrano mettere in atto una strategia di negazione o di repressione delle emozioni negative che inevitabilmente comporta l'utilizzo di un linguaggio limitato o disorganizzato in riferimento agli stati emotivi e una carenza di interazioni verbali relative ai sentimenti (Beeghly e Cicchetti, 1994).

Tali difficoltà nella regolazione delle emozioni risultano essere responsabili, nello sviluppo successivo, della scarsa competenza che i bambini maltrattati hanno nelle relazioni sociali con i coetanei (Cassidy e Kobak, 1988; Radke-Yarrow e Shermann, 1990).

In linea con questa precoce difficoltà non solo nel comprendere, ma anche nell'esprimere ed organizzare le emozioni, si mostra essere uno studio eseguito da Main e Solomon $(1986,1990)$ nel quale viene dimostrato che i neonati maltrattati presentano durante lo sviluppo un pattern disorganizzato/disorientato di attaccamento con il caregiver, secondo un modello di relazione legato alla paura nei confronti di un adulto. Emerge, pertanto, un duplice aspetto legato alle difficoltà derivate dalle precoci esperienze relazionali, che implicano una distorsione del vissuto delle emozioni, come vissuto soggettivo e personale, ma anche una difficoltà cognitiva di interpretazione mentale dell'emozione e dei suoi significati in relazione al sé ed al comportamento dell'altro.

In relazione all'infanzia, un ruolo fondamentale è ricoperto dal modo in cui il bambino si percepisce sulla base dell'interiorizzazione dei comportamenti e delle opinioni degli altri rappresentativi nei suoi confronti, processo che vari autori definiscono "introiezione" (Sullivan, 1953; Freud, 1924; Benjamin, 2003).

Secondo Neff (2003) gli individui che hanno sperimentato un elevato supporto materno e un buon funzionamento familiare durante la crescita presentano valori maggiori di auto-compassione rispetto ad individui cresciuti in famiglie disfunzionali e meno favorevoli. Questi ultimi, al contrario, probabilmente svilupperanno auto-colpevolizzazione e auto-critica. In assenza di auto-compassione, tali introiezioni producono sentimenti di vergogna (Benjamin, 2003; Henry et al., 1990) che possono condurre allo sviluppo di un'immagine del sé sempre più negativa in quanto $\mathrm{i}$ bambini, per giustificare la pericolosità dei genitori e salvarne la relazione, si riputano meritevoli degli abusi che subiscono, giungendo a un senso di disempowerment e impotenza (Herman, 1997).

Durante l'adolescenza, gli abusi subiti e i sentimenti da essi scaturiti ricoprono un ruolo fondamentale nell'ambito dell'autostima. Quest'ultima può essere definita come una forma di apprezzamento personale, rispetto verso il sé (Morganett, 2005), accettazio- ne complessiva della personalità e amore per sé stessi (Adams e Gullota, 1989). Rosenberg. (1965) definisce l'autostima come atteggiamento negativo e positivo dell'individuo nei confronti di sé stesso, risultato dell'autovalutazione dell'individuo. Il verdetto raggiunto in seguito all'autovalutazione indica il livello di autostima, vista come giudizio di dignità legato al concetto di sé. Secondo Pişkin. (2004) il livello di autostima dell'individuo può essere compreso a partire dalla discrepanza presente tra l'immagine di sé (come il soggetto si percepisce) e il suo "io ideale" (come il soggetto vorrebbe essere).

Come evidenziato da Mastronardi (2016), gli adolescenti, durante la loro transizione dalla dipendenza all'indipendenza dai genitori, eseguono non solo una rielaborazione critica dei valori e dei comportamenti dei propri genitori, ma anche continue verifiche del proprio valore in svariate aree personali (scolastica, emozionale, familiare, etc.). I soggetti vittime di maltrattamento, non potendosi basare su un punto fermo quali i genitori, desumono di non aver alcun valore e di essere inadeguati. A seconda del tipo di abuso che il minore ha subito, le sue considerazioni intrapsichiche varieranno: il minore trascurato reputerà di non essere degno dell'amore dei propri genitori e a questa credenza seguirà un sentimento di rabbia congiuntamente al senso di colpa e alla paura; il bambino verbalmente $\mathrm{o}$ fisicamente abusato riterrà che il maltrattamento derivi dal fatto che lui sia sbagliato e che sbagliare sia l'unico modo per avere la loro attenzione; il bambino sessualmente abusato riterrà di essere lui stesso a commettere azioni cattive e segrete, giungendo alla conclusione di essere cattivo, traslando in tal modo le qualità negative tipiche dell'abusante verso il sé (Giusti, 1995).

Il maltrattamento infantile estende i suoi effetti anche all'età adulta, in riferimento alla quale è stato associato a numerosi esiti psicologici e sociali negativi quali maggiore criminalità, livello d'istruzione inferiore, maggior rischio di disturbi psichiatrici, sintomi d'ansia, episodi dissociativi e psicosi (Ferguson e Dacey, 1997; Rowan e Foy, 1993; Valente, 2005; Varese et al., 2012).

Lo studio proposto da Ross, Kaminski e Herrington (2019) ha lo scopo di mettere in luce i meccanismi alla base della depressione negli adulti che sono stati vittime di maltrattamento infantile. Dai risultati emerge il ruolo fondamentale della vergogna, la quale predice la gravità della depressione più fortemente di quanto faccia l'abuso, dimostrando come esplorare ed affrontare i sentimenti di vergogna sia di particolare importanza nel percorso terapeutico di recupero da un maltrattamento passato. Pur mantenendo costanti altre forme di maltrattamento, quello emotivo gioca un ruolo importante nella costruzione del significato delle vittime di abuso riguardo alle avversità che hanno subito in passato. Garbarino (2011) sostiene che siano proprio questi significati a dare al maltrattamento la sua forza distruttiva: la vergogna interferisce con le convinzioni delle vittime riguardanti il cambiamento e la crescita personale, instillando in loro la sensazione di essere intrappolati nella percezione di loro stessi come difettosi. Il miglioramento dell'auto-compassione può avere dei risultati postivi nella riduzione della vergogna e, pertanto, nella riduzione dei sintomi depressivi; può essere dunque il punto d'intervento per ridurre la psicopatologia (Gilbert e Procter, 2006; Johnson e O’Brien, 2013).

\section{Aggressività e maltrattamento infantile}

Baron e Richardson (1994) definiscono l'aggressione come un qualsiasi comportamento volto a recare danno o ferire un altro essere vivente che è motivato ad evitare tale trattamento, intendendo con danno una qualunque ferita fisica/psicologica, la compro- 
missione delle relazioni sociali, e/o la distruzione di beni posseduti dal target.

Questa definizione comporta tre fondamentali implicazioni, evidenziate da Krahé (2015):

1) l'aggressione è definita non dalle sue conseguenze (effettivo verificarsi del danno o della ferita), ma dalle sue motivazioni sottostanti (volontà e intenzione di danneggiare o ferire) e non necessita il concreto danneggiamento della vittima;

2) l'intenzione del soggetto implica la capacità di comprendere che il comportamento presenta le potenzialità di danneggiare o ferire; nel caso in cui le azioni che danneggiano non siano caratterizzate da questa consapevolezza, non rappresentano comportamenti aggressivi;

3) intendere l'aggressione come un comportamento che la vittima vuole evitare esclude le azioni dannose che vengono eseguite con il consenso del target.

La definizione include svariate sottocategorie, quali le aggressioni fisiche e verbali, spontanee o di reazione, individuali o di gruppo.

Sulla base dei differenti approcci teorici utilizzati vengono eseguite ulteriori distinzioni, come quella eseguita tra l'aggressione diretta/manifesta, che implica un confronto fisico tra aggressore e target, e quella indiretta/relazionale, che mina le relazioni sociali del target attraverso la calunnia o la diffamazione, vista come una strategia alternativa ai costi elevati dell'aggressività diretta (Crick e Grotpeter, 1995).

Altra fondamentale distinzione opera un rimando alle motivazioni sottostanti alle aggressioni: strumentali oppure ostili (anche dette affettive o rabbiose). Le aggressioni strumentali vengono messe in atto con il fine di raggiungere determinati obiettivi (ad es.: prendere un soggetto in ostaggio con l'obiettivo di ottenere la somma del riscatto), per cui il comportamento aggressivo si pone come un mezzo possibile, ed eventuali danni a persone o cose sono considerati come meri effetti collaterali inevitabili. Al contrario, le aggressioni ostili sono mosse dal desiderio del soggetto agente di esprimere e mostrare i propri sentimenti negativi (es: rabbia) e l'obiettivo primario è quello di recare un danno al target (Berkowitz, 1993).

La distinzione principalmente analizzata in letteratura vede l'aggressività proattiva contrapporsi a quella reattiva. La prima trova le sue basi nella teoria dell'apprendimento social-cognitivo di Bandura, che definisce l'aggressività generale come un prodotto di elevata auto-efficacia per comportamenti aggressivi generali, ponendo l'enfasi su risultati desiderabili e ricompense per il comportamento aggressivo (Crick e Dodge, 1996). La seconda presenta la sua base teorica nel modello frustrazione-aggressività di Berkowitz, il cui modello evidenzia il ruolo fondamentale degli stati emotivi negativi, che attivano risposte affettive e cognitive negative accrescendo la probabilità che intenzioni aggressive si tramutino in comportamento. L'aggressione reattiva è associata al pregiudizio dell'attribuzione ostile (Dodge et al., 1990), e gli individui reattivamente aggressivi mostrano delle difficoltà nel codificare e interpretare gli spunti sociali, visti come deficit nell'elaborazione dell'informazione sociale.

L'aggressività proattiva è motivata da ricompense esterne, dal bisogno di raggiungere degli obiettivi personali attraverso uno scarso coinvolgimento della sfera emotiva (Glenn e Raine, 2009), che viene invece comunemente attivata nelle altre forme di aggressione non psicopatiche (Blair, 2010; Raine et al., 2006). Costituisce un'aggressione fredda, premeditata e manipolatrice (Blais, Solodukhin e Forth, 2014), associata a tratti antisociali della personalità (Fite et al., 2010). Gli individui che esercitano aggressività proattiva sono organizzati e predatori, spesso possiedono un fascino superficiale che funziona nel breve periodo, ma mantengo- no poche o nessuna relazione duratura a causa delle loro scarse capacità interpersonali (Raine et al., 2006).

L'aggressività reattiva, al contrario, è calda, impulsiva ed emotivamente guidata (Blais, Solodukhin, e Forth, 2014), spesso associata ad abusi durante l'infanzia e difficoltà nel regolare le emozioni (Marsee e Frick, 2007). Può comportare comportamenti intenzionalmente dannosi per ritorsione o difesa contro l'individuo percepito come una minaccia (Crick e Dodge, 1996). L'aggressione reattiva è stata collegata ad un aumento dei livelli di tristezza, depressione e infelicità (Shackman e Pollak, 2014). Mentre il meccanismo specifico che collega l'aggressività reattiva con l'emotività negativa, che può portare a un aumento del rischio di isolamento sociale, rimane poco chiaro, si ipotizza che il rifiuto sociale possa svolgere un ruolo importante nell'interazione (Fite et al., 2010). Un aspetto chiave dell'aggressione reattiva è la differenziazione tra una minaccia o un insulto percepito e una minaccia o un insulto legittimo. Gli individui reattivamente aggressivi sono incapaci di discernere tra i due e spesso interpretano erroneamente alcune situazioni portando a una conseguente aggressione impulsiva ed esplosiva (Raine et al., 2006; Rappaport e Thomas, 2004).

È presente in letteratura un considerevole corpus di ricerche che dimostrano saldamente l'associazione tra maltrattamenti infantili ed esiti disadattivi in età adulta, come aggressività, comportamenti antisociali e criminalità, nonostante l'esatto meccanismo che conduce a questi esiti negativi non sia ad oggi completamente chiaro (Dotson, 2016).

Uno studio condotto da Shackman e Pollak (2014) ha dimostrato che gli individui che hanno subito abusi fisici durante l'infanzia hanno riportato un aumento del tasso di problemi di esternalizzazione caratterizzati da aggressività reattiva, accompagnata da un'erronea interpretazione degli spunti sociali, una regolazione inappropriata delle emozioni, un aumento dell'eccitazione simpatica e dell'impulsività e una maggiore tendenza a rispondere con comportamenti aggressivi (Dodge et al., 1995; Shackman e Pollak, 2014).

Allen (2011), in uno studio sugli abusi psicologici infantili, ha dimostrato che l'abuso verbale e psicologico comporta un significativo aumento del punteggio sulle scale di misura dell'aggressione. Tuttavia, è necessario considerare che la ricerca che isola una particolare forma di maltrattamento può essere problematica, visto l'elevato grado di co-occorrenza tra i vari tipi di maltrattamento (Allen, 2011; Higgins e McCabe, 2000).

Caspi et al. (2002) indicano i maltrattamenti infantili come fattori di rischio universale per i comportamenti aggressivi e antisociali, provocando un aumento del rischio di atti criminali successivi fino al 50\%, anche se la maggior parte dei bambini maltrattati non diventa criminale. Il loro studio si concentrò sull'interazione tra geni ed ambiente, esaminando gli effetti del gene monoamino ossidasi A (MAOA) su individui abusati in confronto con individui non abusati. I risultati dello studio suggeriscono che il gene MAOA sia positivamente associato all'abuso infantile e successivamente alla sintomatologia aggressiva.

Lo studio di Dotson (2016) ha dimostrato che gli abusi fisici precoci (subiti tra 0 e 9 anni), se estremi, sono predittori statisticamente significativi dell'aggressività reattiva-manifesta. Non è stata invece riscontrata una relazione significativa tra gli abusi fisici precoci e l'aggressività relazionale, né di tipo reattivo, né di tipo proattivo. Nel caso di abusi fisici tardivi (subiti tra i 10 e i 18 anni) è emersa una significativa differenza di genere in relazione all'aggressività proattiva-manifesta, con punteggi di aggressività significativamente più alti negli uomini. L'abuso psicologico tardivo si è dimostrato un predittore di aggressività reattiva-manifesta più attendibile rispetto all'abuso fisico sia precoce che tardivo, con una influenza maggiore negli uomini rispetto alle donne. Considerando i punteggi per gli abusi psicologici estremi, è stata individuata una 
correlazione significativa anche con l'aggressività proattiva-manifesta e l'aggressività proattiva-relazionale.

\section{Serial killer, spree killer e mass murderer}

Il serial killer, secondo la definizione di Douglas e Olshaker (1999), è un aggressore che esegue almeno tre omicidi in differenti occasioni, intervallati da un periodo di "cooling-off", ovvero di raffreddamento emotivo, che può avere una durata variabile da pochi giorni a diversi anni, rendendo ogni omicidio emozionalmente distinto e separato.

Mastronardi e Palermo (1995), riprendendo la classificazione eseguita dalla Sezione di Scienza del Comportamento dell'FBI (1990), propongono le seguenti tipologie di assassino seriale:

1) il serial killer visionario: agisce seguendo delle visioni (spesso di presenze interne identificate come entità o demoni) o delle voci (sostengono che la voce di Dio/Satana li incoraggi al massacro) allucinate che lo spingono ad uccidere, le sue azioni sono conseguenze di disturbi mentali come il disturbo da delirio paranoideo, che potrebbe portare a un omicidio pianificato, o la schizofrenia paranoide, che probabilmente produrrà un omicidio scarsamente organizzato;

2) il serial killer missionario: agisce nella ferma convinzione di dover ripulire il mondo da soggetti per lui indesiderabili e indegni (spesso prostitute o vagabondi) e agire così per il bene della società (per questo motivo non prova rimorso), quasi mai è affetto da psicosi ma spesso potrebbero verificarsi delle dispercezioni paranoidi utili a consolidare le sue credenze;

3) il serial killer edonista: agisce con il mero scopo di trarne piacere e raggiungere il cosiddetto "orgasmo omicidiario";

4) il serial killer orientato al controllo del potere: agisce con l'obiettivo di esercitare il controllo totale su un altro individuo e godere del potere supremo di decidere del suo destino;

5) il serial killer lussurioso: agisce per colmare l'angoscia che deriva dai suoi profondi conflitti inconsci e preconsci, con un comportamento ossessivo-compulsivo, libidinoso, spesso ritualistico (fantastica l'omicidio, individua la vittima, esegue il delitto e infine ricolloca i resti della vittima nella fase totemica), provando non solo senso di potere, ma anche gratificazione sessuale.

Fox e Levin (1994) hanno eseguito una classificazione differente incentrandosi sulle motivazioni alla base dei loro omicidi, identificando 5 distinte categorie:

1) il killer del potere: tortura e uccide sadicamente per soddisfare il suo bisogno di controllo e dominio;

2) il killer della vendetta: uccide le donne che rievocano la propria madre che lo ha maltrattato durante l'infanzia;

3) il killer della lealtà: è inserito all'interno di una "squadra" di assassini che utilizzano l'omicidio come un rituale che dimostri l'impegno dei vari componenti;

4) il killer del profitto: avvelena a morte i coniugi per riscuotere la loro assicurazione sulla vita;

5) il killer del terrore: è profondamente paranoico e utilizza delle bombe per avvertire il mondo di una condanna imminente.

Ulteriore tipologia individuata è rappresentata dal serial killer sadico, che esercita un dominio psicologico e un controllo fisico sulle vittime, le umilia, esegue su esse violenza sessuale sadica, senza provare alcun sentimento di rimorso, colpa o vergogna per le proprie azioni (Palermo e Mastronardi, 2005). Sulla base delle teorie sviluppate da Freud (1960) il sadismo sarebbe conseguenza di un arresto dello sviluppo psicosessuale dell'individuo allo stadio chiamato "sadico-anale" o da una regressione nevrotica a tale sta- dio, e rappresenterebbe la manifestazione esteriore dell'istinto di morte. Spesso, queste tensioni sadiche si sviluppano in soggetti che sentono di aver ricevuto, durante la propria infanzia, trattamenti sadici, innescando il procedimento dell'identificazione con l'aggressore nel tentativo di convertire l'esperienza passiva subita in un'esperienza attiva inflitta. Il serial killer sadico mostra un frequente utilizzo del sadistic overkilling, ovvero un esasperato accanimento sulla vittima, spesso eseguito con tagli alla gola, coltellate in pancia, mutilazioni, squarcio del petto, espianto di organi, esposizione teatrale di questi o dell'arma nella scena del crimine (Palermo e Mastronardi, 2005).

Il mass murderer uccide, o tenta di uccidere, nello stesso momento e nello stesso luogo diverse persone, che non sono da lui conosciute ma identificate in modo casuale. Il Federal Bureau of Investigation, al fine di comprendere un omicida in tale categoria, ritiene ora necessarie almeno tre vittime contestuali (Douglas et al., 2008), come precedentemente sostenuto da Mastronardi e Palermo (2000). In questa categoria non vengono incluse le stragi belliche, terroristiche o mafiose, in quanto legate a motivazione estrinseche, non intrinseche come quelle dell'assassino di massa.

Palermo e Mastronardi (2005) hanno proposto la principale distinzione eseguita all'interno di questa categoria, che vede una ripartizione tra classic mass murderer e family mass murderer. L'omicida di massa classico rivolge la sua aggressività omicidiaria verso soggetti sconosciuti ed estranei alle sue problematiche ma che vengono dal soggetto indentificati come simboli della collettività da punire, ovvero la società, da cui è convinto di aver subito grandissimi torti (spesso è un soggetto paranoico o schizofrenico paranoide). A differenza del serial killer, non è interessato al contatto fisico, e utilizza prevalentemente armi da fuoco con lo scopo di causare più morti possibili durante la strage, al termine della quale spesso si toglie la vita o viene ucciso delle forze dell'ordine (Palermo e Mastronardi, 2005). L'omicida di massa familiare dirige la propria aggressività verso la propria famiglia, coinvolgendo spesso altri parenti, vicini di casa o ignari passanti che si trovano nel luogo della strage. Comunemente, il soggetto in seguito alla strage si suicida, per questo motivo viene spesso definito mass murderer/suicide. In tal caso, il soggetto aveva precedentemente stabilito di porre fine alla sua vita, ma sceglie di eseguirlo con una modalità eclatante, coinvolgendo il resto della famiglia per non abbandonarle in questo mondo crudele. Questi soggetti sono spesso affetti da disturbi mentali o della personalità, quali depressione maggiore, sindromi persecutorie o psicosi allucinatorie con deliri di grandezza, ma talvolta eseguono queste azioni d'impulso o per motivi passionali. Il mass murderer solitamente conduce una vita solitaria, dimostra difficoltà relazionale e diffidenza a causa della sua infanzia, spesso infelice, e agisce per rivendicazione sociale e politica (Palermoe Mastronardi, 2005).

Altra classificazione proposta da Palermo e Mastronardi (2005) si basa sulle motivazioni alla base della strage, e prevede la tripartizione in: mass murderer patologici propriamente detti, mass murderer strumentali e mass murderer passionali.

I primi includono:

1) soggetti con disturbi depressivi: guidati da deliri di colpa o indegnità, uccidono quando la depressione raggiunge livelli esasperati e si convincono che non vi sia più alcuna via di fuga, solitamente è presente un evento scatenante anche di lieve importanza che porta il soggetto alla scelta del suicidio, portando con sé la propria famiglia per risparmiar loro il dolore della sua perdita, spesso i figli vengono uccisi perché percepiti come parte del sé;

2) bugiardi patologici: affetti da disturbi di personalità e talvolta con nuclei psicotici, uccidono per evitare che le loro bugie vengano scoperte, soprattutto dai propri genitori che sarebbero 
delusi dalla realtà dei fatti, spesso la causa scatenante è la necessità di un'immediata dimostrazione delle proprie dichiarazioni;

3) soggetti con disturbi mentali: spesso affetti da disturbo paranoide di personalità o da schizofrenia, uccidono perché la morte viene ritenuta l'unica soluzione possibile al male, che viene avvertito come eterno.

Tra gli strumentali vi troviamo:

1) i libertari: ricercano la libertà dall'oppressione genitoriale;

2) i tossicomani: uccidono abitualmente in crisi d'astinenza se vengono loro negati i soldi necessari per l'acquisto della droga;

3) gli ereditieri: uccidono per trarre un guadagno economico anche se non ingente.

Nella categoria dei mass murderer passionali identifichiamo:

1) i gelosi: uccidono per gelosia in riferimento ad un fatto realmente accaduto o immaginato, nella convinzione di poter disporre della vita e della morte della loro famiglia, vista come res propria;

2) i vendicativi/persecutori: uccidono per vendicarsi di un torto reale o immaginato, non accettano la fine della relazione o l'abbandono del partner e in seguito diventano degli stalker, eseguendo terrorismo psicologico verso la vittima, atti vandalici verso le sue proprietà fino ad arrivare alla strage, ben pianificata, per colmare la paura che la vittima trovi un nuovo partner;

3) i litigiosi: uccidono sotto la forte spinta emotiva in seguito a una lite familiare, a seconda dei motivi per cui scaturisce la lite possono presentarsi delle sovrapposizioni con categorie precedentemente citate (es: tossicodipendenti/litigiosi).

Lo spree killer viene definito come l'autore di almeno due delitti eseguiti in tempi e luoghi differenti ma contigui, come se il soggetto si conformasse ad una sola spinta distruttiva, innescata dalla medesima causa scatenante (Palermo e Mastronardi, 2005). Spesso non conosce le sue vittime e non rimuove le tracce dei suoi omicidi, giungendo ad essere facilmente identificato e, quasi sempre, al termine del suo "spree" commette il suicidio.

Quella dello spree killer è una categoria criminologica controversa, che non vede un'interpretazione univoca della letteratura. Newton (1990) sostiene che questa categoria non presenti una propria autonomia, ma rientri nella categoria più ampia dei serial killer, con la particolarità di un minimo o assente periodo di raffreddamento emotivo tra i vari delitti. Interpretazione non condivisa da Palermo e Mastronardi (2005) secondo cui nel serial killer è presente l'intenzione di continuare a ripetere nel tempo le azioni che lo hanno gratificato, che vengono di conseguenza eseguite con prudenza per evitare di lasciare tracce o essere identificato; nello spree killer quest'intenzione è assente, esegue le sue azioni come se tutto finisse in quel momento, non avvertendo l'esigenza di occultare le prove del suo agire. Le vittime dello spree killer sono sempre diverse, come diversi sono i luoghi in cui agisce, è inarrestabile e imprevedibile in quanto guidato da spinte altamente emotive (rabbia/vendetta), i suoi omicidi ravvicinati causano panico tra la popolazione e ricevono, per questo motivo, ampissima attenzione mediatica. Sono da considerare killer narcisisti, emotivamente e socialmente instabili e disorganizzati, mossi da un forte senso di rifiuto e vendetta (Palermo e Mastronardi, 2005).

\section{Assassini multipli e maltrattamento infantile}

Il serial killer è la tipologia di assassino multiplo su cui si è concentrata la percentuale maggiore degli studi effettuati, giungendo a diverse proposte in riferimento allo sviluppo della sua personalità.
Secondo il trauma-control model, proposto da Hickey (1997), un soggetto sopprime a livello inconscio determinati eventi destabilizzanti sperimentati durante l'infanzia o l'adolescenza (abusi sessuali/fisici/psicologici, morte di un genitore, etc.). Questi eventi vengono vissuti anche da soggetti che non diventano serial killer, per questo motivo viene ipotizzata una predisposizione (psicologi$\mathrm{ca} /$ biologica/sociologica) o vulnerabilità significativa, che in aggiunta ai traumi infantili producono nel soggetto sentimenti quali rifiuto, sfiducia, confusione e ansia. La memoria degli eventi traumatici sperimentati si scinde dalla regolare personalità del soggetto, ma potrebbe eventualmente riemergere, specialmente (ma non esclusivamente) quando intervengo dei facilitatori, quali droghe, alcol e pornografia, che riducono le inibizioni e possono condurre all'uccisione. Hickey sostiene che l'omicidio aiuti il soggetto a costruire una maschera di autocontrollo e fiducia, recuperando l'equilibrio psicologico che è stato lui tolto dalle persone autorevoli della sua vita.

Holmes et al. (1999), con l'ipotesi della fractured identity syndrome, sostengono l'idea di Hickey secondo cui traumi nell'infanzia e nell'adolescenza possano provocare una piccola fessura nella personalità del soggetto, sostenendo che i traumi successivi potrebbero causare l'allargamento della fessura e una conseguente frattura della personalità, ma non una sua distruzione totale.

Un recente studio (Marono et al., 2020) ha indagato gli effetti di tre differenti tipi di abuso infantile (fisico, psicologico e sessuale) su quattro tipologie di serial killer identificate sulla base della motivazione (lussuria, rabbia, potere, profitto finanziario). Lo studio ha preso in considerazione un campione maschile di 233 serial killer con una storia documentata di abusi infantili; dai risultati è emerso che i metodi di uccisione più violenti (mutilazione, tortura, etc.) vengono praticati da coloro che nella prima infanzia sono stati abusati sessualmente o psicologicamente. Coloro che hanno subito abusi sessuali raramente mostrano prove di overkilling e tendono ad eseguire gli omicidi in modo rapido, a differenza di coloro che hanno subito un abuso físico o psicologico, in cui l'overkilling è stato riscontrato con maggiore frequenza. Questo può essere compreso con un riferimento alla profonda rabbia e al senso di colpa che caratterizza coloro che hanno subito abusi sessuali: questi sentimenti causano una rapida uccisione delle loro vittime e sensi di colpa o rimorso in seguito all'omicidio, che rendono improbabile un eccesso nell'uccisione (Briere e Elliott, 1994; Wyatt e Newcomb, 1990). I serial killer classificati come "motivati dal potere" hanno compiuto rapidamente tutti gli omicidi registrati e non vi erano prove di torture, mutilazioni o overkilling. La ragione potrebbe derivare dal bisogno di controllare la vittima e affermare il proprio dominio su essa (Canter e Wentink, 2004; Holmes e Holmes, 1998). In coloro che sono stati classificati nella tipologia della lussuria è stata comunemente riscontrata la pratica del sesso post-mortem, indipendentemente dal tipo di abuso subito da bambino, e in nessuno dei casi è stata riscontrata alcuna prova di overkilling, sebbene la tortura fosse comunemente usata.

È importante sottolineare che l'esperienza di abuso sessuale, isolata o vissuta insieme all'abuso fisico e/o psicologico, ha portato alla mutilazione o alla tortura della vittima. Allo stesso modo, gli individui che sono stati classificati nella tipologia di lussuria/stupro presentano maggiori probabilità di torturare o mutilare le loro vittime, suggerendo una correlazione tra il comportamento sessuale e la necessità di infliggere dolore.

Lo studio di Abe (2015) ha analizzato il vissuto infantile di 28 mass murderer con la finalità di identificare dei modelli ricorrenti e di comprendere se questi siano orientati verso la negligenza, verso l'abuso o ad entrambi.

Sono state identificate nove tipologie di vissuto ambientale che potessero essere collegate ai mass murderer: bambino lasciato incu- 
stodito per disinteresse/ragioni finanziarie/interesse personale (Thomas Hamilton, Ronald Simmons, Joseph Wesbecker, James Huberty); bambino intenzionalmente escluso dalla famiglia (One Goh, James Ruppert, Eric Borel, Andrew Kohoe); negligenza postnatale/nessun contatto fisico a causa di peculiare personalità/disturbo mentale della madre (Martin Bryant, Anders Breivik, Howard Unruh, Adam Lanza, Dylan Klebold); comunicazione anormale con e tra i genitori dovuta alla loro relazione/personalità fredda (James Holmes, Seung-Hui Cho); genitore/i molto rigido/i (Baruch Goldstein, Nidal Hasan); madre iperprotettiva (Michael Ryan); impossibilità di esprimere reali sentimenti a causa di una famiglia insicura/esigente (Timothy McVeigh, Charles Whitman, Julian Knight, Jacob Roberts, Mark Lepine, George Hennard, Eric Harris); psicopatico secondario altamente intossicato (Richard Speck, George Banks); schizofrenia indotta dalla droga (Jared Loughner).

Dai risultati è emerso che quasi un terzo del campione è stato isolato o vittima di bullismo a scuola. Per quanto riguarda il tipo di maltrattamento, la trascuratezza (dal tipo 1 al tipo 4) è la tipologia decisamente prevalente. Alcuni soggetti non presentavano un vissuto chiaro ed è stato specificato il motivo della loro categorizzazione.

Un testimone non identificato ha riferito che Lanza, che aveva la sindrome di Asperger, pensava che sua madre amasse i suoi studenti alla scuola elementare Sandy Hook più di lui, e per questo motivo la odiava. Ipotesi supportata dai quattro colpi alla testa inferti alla madre prima della strage alla Sandy Hook. Lui e sua madre comunicavano esclusivamente via e-mail e lei per due giorni prima della sparatoria avrebbe soggiornato in un hotel come parte di un esperimento per lasciare che diventasse indipendente, e questi comportamenti rigidi e severi sono probabilmente iniziati in tenera età. Abe e Kato (2007) suggeriscono che i fattori ambientali potrebbero facilitare i sintomi della sindrome di Asperger, come dissociazione, ansia, depressione, deliri persecutori e comportamento antisociale. Pertanto, Lanza è stato inserito nella categoria "abbandono postnatale / nessun contatto físico".

Il reale vissuto di Eric Harris e Dylan Klebold. autori del massacro della Columbine High School, sembrano poco chiari. Alcuni ricercatori sottolineano che erano bulli piuttosto che bullizzati. Eric, che era considerato il principale trasgressore dei due, si definiva un "Dio della tristezza", e una volta disse: "Vi odio gente per avermi lasciato fuori da così tante cose divertenti." Allo stesso modo Dylan scrisse nel suo diario: "Sono sempre stato odiato da tutti e da tutto" (Toppo, 2009). Dylan, a causa dell'orientamento accademico della madre, ha frequentato uno speciale corso d'élite durante gli studi elementari, e in seguito ha sviluppato una notevole irascibilità. Il padre di Eric era nell'esercito, non tollerava comportamenti scorretti in casa e la punizione era rapida e dura. Intorno ai 12 anni, Eric ha iniziato a mostrare episodi di piromania e atti vandalici (Cullen, 2009). Eric stesso, in seguito, suggerì di essere stato costretto a fingere di essere un bambino inobbediente sotto la pressione inespressa della famiglia. Considerando queste informazioni, Eric è stato inserito nella categoria "impossibile esprimere reali sentimenti " e Dylan nella categoria "abbandono postnatale/nessun contatto fisico".

Alcuni ricercatori affermano che Seung-Hui Cho, autore della strage al Virginia Tech, parlava con la sua famiglia settimanalmente e che questa era parte integrante della sua vita. Tuttavia, secondo uno psichiatra infantile che ha seguito la famiglia di Cho, lui non ha parlato molto della sua vita, neanche con sua sorella, che era presumibilmente una delle pochissime persone con cui Cho si sentiva a suo agio. Il suo compagno di stanza del dormitorio ha sostenuto che era presente un'insolita freddezza tra Cho e i suoi genitori, e interazioni familiari anormali. A Cho è stato diagnosticato un mutismo selettivo, quindi, è stato posto sotto "comunicazione anormale".
Richard Speck, autore della strage al South Chicago Community Hospital, in seguito alla morte di suo padre, al quale era molto legato, è stato abusato psicologicamente dal suo patrigno. George Banks, responsabile delle "1982 Wilkes-Barre shootings" è stato costantemente vittima di bullismo a causa del colore della sua pelle. Entrambi sembravano aver sviluppato affetti normali: Speck aveva anche sorelle premurose, mentre Banks aveva uno stretto rapporto con sua madre e alcuni amici. Tuttavia, a causa del loro ambiente avverso, hanno sviluppato una psicopatia secondaria, causando numerose risse e commettendo reati minori ripetuti. Al momento delle stragi, Speck era sotto effetto sia di alcol che di droghe, e Banks di una miscela di farmaci da prescrizione e gin. In entrambi i casi, si è ritenuto che l'ingestione delle sostanze abbia svolto un ruolo importante nell'innescare l'omicidio di massa. Pertanto, Speck e Banks sono stati etichettati come "psicopatici secondari altamente intossicati”, sebbene condividano caratteristiche con altre categorie.

Jared Loughner, responsabile del "2011 Tucson shooting”, era molto riservato, così come la sua famiglia. La ragazza di Loughner ha affermato che i suoi genitori non l'hanno mai lasciata entrare in casa e che raramente hanno agito come figure genitoriali. In seguito alla fine della relazione con la ragazza c'è stato un cambiamento nella sua personalità: Loughner iniziò ad abusare di alcol e droghe e la sua vita iniziò a disfarsi. Pertanto, sebbene condivida i tratti con la categoria "intenzionalmente escluso dalla famiglia", considerando che il suo drastico cambiamento di personalità è avvenuto dopo l'abuso di droghe, è stato inserito nella categoria "schizofrenia indotta dalla droga".

Anche le tipologie 5-6-7 ("genitore/i molto rigido/i", "madre iperprotettiva" e "impossibilità di esprimere reali sentimenti") possono essere ricondotte ad un ambiente negligente, in quanto non rispettano le necessità e i bisogni del bambino. È possibile che le influenze negative degli ambienti negligenti abbiano causato ai mass murderer difficoltà con le relazioni, che hanno causato il loro successivo isolamento e le esperienze di bullismo (Horwitz et al., 2001). Si ipotizza che i bambini trascurati sviluppino un modello operativo interno distorto. Nel peggiore dei casi, possono sviluppare un disturbo dell'attaccamento reattivo o un cervello neurologicamente compromesso, che si traduce nella loro preoccupazione per le dinamiche relazionali negative, l'isolamento psicologico e atteggiamenti evitanti verso gli altri (Hildyard e Wolfe, 2002; Glaser, 2000). Potrebbe essere questo stato mentale che in seguito porta ai loro impulsi violenti, che sono rappresentati da invidia patologica, fantasie di vendetta e rabbia inspiegabile (Hemple et al., 1999; Knoll, 2010a; Knoll, 2010b). Dunque, l'aberrazione genetica non è l'unico fattore che porta alla violenza; fattori ambientali come le avversità vissute durante l'infanzia svolgono un ruolo significativo nello sviluppo del comportamento violento (Reif et al., 2007).

Il recente studio di Abe (2019), ha analizzato tre fattori che conducono a una delle tre tipologie di assassino multiplo: vissuto infantile, motivazioni che spingono il soggetto ad agire e disponibilità di interazioni umane durante l'infanzia (Tab. 1). Nello studio, le tre tipologie vengono poste all'interno di un continuum, in cui serial killer e mass murderer sono posizionati a poli opposti e tra $i$ due sono inseriti gli spree killer.

Le posizioni di serial killer e mass murderer sono opposte a causa della natura contrastiva del loro background. I mass murderer sono statisticamente stati vittime di pratiche trascuranti come l'abbandono, il controllo mentale (manipolazione del bambino affinché non soddisfi i propri bisogni ma quelli dei genitori), la rigida educazione del bambino e l'iperprotezione (Abe, 2015). I serial killer, al contrario, hanno subito pratiche abusive di educazione come abusi fisici, sessuali e/o psicologici, tra cui la negazio- 
Tab. 1. Fattori che conducono a una delle tre tipologie di assassino multiplo, secondo lo studio di Abe (2019).

\begin{tabular}{|c|c|}
\hline Type of murder & Mass murder — Spree murder_—Serial murder \\
\hline Background & $\varphi(\mathrm{ng} / \mathrm{mc} / \mathrm{rg} / \mathrm{op})-$ Continuum $-\downarrow(\mathrm{ab} / \mathrm{ex} / \mathrm{man})$ \\
\hline Interaction & Low- High- \\
\hline Motive & Mission_-Outburst/Cannot Stop___easant sensation \\
\hline Onset & Adolescence $\rightarrow$ Years Later \\
\hline
\end{tabular}

ne dell'identità e dell'esistenza, che producono o accrescono il loro desiderio di ferire o distruggere gli altri (Hale, 1993; Vandello et al., 2008). Dunque, i serial killer, durante la loro infanzia, hanno affrontato forze esterne innaturali, mentre i mass murderer non hanno goduto delle forze umane necessarie per la crescita e la vita sociale (Hildyard e Wolfe, 2002). Agiscono anche per motivazioni differenti: i serial killer in linea generale commettono gli omicidi per vivere le piacevoli sensazioni in loro provocate, i mass murderer agiscono spinti da un bisogno di giustizia sociale (Knoll, 2010a). Gli spree killer possono provenire da background familiari altamente trascuranti con relazioni umane disponibili, oppure da background altamente abusivi con relazioni umane disponibili. Nel primo caso, i soggetti tentano di inibire la loro spinta omicida senza successo, a causa dei desideri sviluppati per colmare il vuoto della negligenza materna durante l'infanzia (Macdonald, 1963). $\mathrm{Nel}$ secondo caso, in seguito al primo omicidio, il soggetto, in preda all'eccitazione, esplode in una catena di furia omicida fin quando non viene fermato da qualche forza esterna. Negli spree killer non è presente una deviazione della personalità profonda quanto quella dei serial killer e dei mass murderer, che non hanno sperimentato relazioni umane, per questo motivo provano spesso forti sensi di colpa. Essendo le tre tipologie poste in un continuum, alcuni spree killer possono mostrare delle caratteristiche molto simili a quelle che contraddistinguono i serial killer, oppure, viceversa, a quelle che contraddistinguono i mass murderer.

Queste tre tipologie sono accomunate in quanto forme di violenza vendicativa distorta, non diretta nei confronti degli originali autori dell'abuso o della negligenza, ma rivolta ad obiettivi secondari spesso totalmente estranei alle vicende del soggetto. I tre tipi di assassini iniziano a mostrare dei "sintomi" durante l'adolescenza (Vandello et al., 2008), accompagnata da stravolgimento ormonale e maturazione psicologica (MacCulloch, 1985). La maggior parte giustificherà gli omicidi incolpando le vittime o appoggiando religioni sataniche o filosofie radicali, ad eccezione di alcuni spree killer, e raramente anche serial killer e mass murderer che hanno sviluppato delle emozioni considerabili normali (Abe, 2017).

\section{Bibliografia}

Abe K (2015). Nine types of childhood environment that actually produced mass murderers based on the information in lay literature and on the internet. European Journal of Academic Essays 2(7):45-55.

Abe K (2017). What is a serial killer? What is a mass murderer? How do they differ? European Journal of Academic Essays 4(4):187-198.

Abe K (2019). Serial killers, mass murderers, and spree killers: Three factors decide the murder type on the same continuum, in International Journal of Social Science and Humanities Research, 7(2):137-141.

Abe T, Kato S (2007). Environmental factors in Asperger syn- drome. Nihon Rinsho. Japanese Journal of Clinical Medicine 65(3):439-442.

Adams GR, Gullota T (1989). Adolescent life experience. Belmont: Brooks/Cole

Allen B (2011). Childhood psychological abuse and adult aggression: The mediating role of self-capacities. Journal of Interpersonal Violence, 26(10):2093-2110.

American Psychiatric Association (a cura di) (2013). DSM 5 (tr. it)., Manuale diagnostico e statistico dei disturbi mentali, Milano, Raffaello Cortina, 2014.

Baker AJL, Verrocchio MC (2016). Exposure to parental alienation and subsequent anxiety and depression in Italian adults. American Journal of Family Therapy 44(5):255-271.

Baron RA, Richardson DR (1994). Human Aggression. New York: Plenum.

Beeghly M, Cicchetti D (1994). Child maltreatment, attachment and the self styme: emergence of an internal state lexicon in toddlers at high social risk. Development and Psychopathology 6:5-30.

Benjamin LS (2003). Interpersonal reconstructive therapy: Promoting change in nonresponders. New York: Guilford.

Berkowitz L (1993). Aggression: Its causes, consequences and control. New York: McGraw-Hill, pp. 103-133.

Blair RR (2010). Psychopathy, frustration, and reactive aggression: The role of ventromedial prefrontal cortex. British Journal of Psychology 101:383-399.

Blais J, Solodukhin E, Forth AE (2014). A meta-analysis exploring the relationship between psychopathy and instrumental versus reactive violence. Criminal Justice and Behavior 41:797-821.

Briere JN, Elliott DM (1994). Immediate and long-term impacts of child sexual abuse. Future of Children 4(2):54.

Canter DV, Wentink N (2004). An empirical test of Holmes and Holmes's serial murder typology. Criminal Justice and Behavior 31(4):489-515.

Carlson J, Cicchetti D, Barnett D, Brawnwald KG (1989). Finding order in disorganization: lessons from research on maltreated infants' attachments to their caregivers. In: D. Cicchetti, J. Carlson (a cura di) (1989), Child maltreatment: theory and research on the causes and consequences of child abuse and neglect. Cambridge: Cambridge University Press, pp. 494-528.

Caspi A, McClay J, Moffitt ET, et al. (2002). Role of genotype in the cycle of violence in maltreated children. Science 297(5582):851-854.

Cassidy J, Kobak RR (1988). Avoidance and its relationships to other difensive processes. In: J. Belsky, T. Nezworsky, Clinical implications of attachment. Hillsdale: Erlbaum, pp. 300-323.

Coster W, Gersten MS, Beeghly M, Cicchetti D (1989). Communicative functioning in maltreated toddlers. Developmental Psychology 25:1020-1029.

Crick NR, Dodge KA (1996). Social information-processing mechanisms on reactive and proactive aggression. Child Development 67:993-1002.

Crick NR, Grotpeter JK (1995). Relational aggression, gender, and 
social- psychological adjustment. Child Development 66:710722.

Cullen D (2009). Columbine. New York: Twelve.

Di Blasio P, Rossi G (2004). Trascuratezza, maltrattamento e abuso in danno dell'infanzia: Servizi e Centri presenti in Regione Lombardia. Report di ricerca, Università Cattolica del sacro Cuore di Milano e Regione Lombardia, Milano.

Di Blasio P (2000). Psicologia del bambino maltrattato. Bologna: Il Mulino.

Dodge KA, Price JM, Bachorowski JA, Newman JP (1990). Hostile attributional biases in severely aggressive adolescents. Journal of Abnormal Psychology 99:385-392.

Dotson E (2016). Aggressive and depressed: long-term outcomes of childhood maltreatment. EWU Masters Thesis Collection.

Douglas J, Burgess AW, Burgess AG, Ressler RK (2008). Crime Classification Manual. Il manuale dell'FBI sulla classificazione e investigazione dei crimini violenti. Torino: Centro Scientifico.

Douglas JE, Olshaker M (1999). The Anatomy of Motive. New York: Scribner/Lisa Drew.

Emiliani F, Simonelli A (1997). Psicopatologia evolutiva e maltrattamento infantile. Psicologia clinica dello sviluppo 3:323-362

Federal Bureau Investigation (1990). Criminal Investigative Analysis: Sexual Homicide. Quantico: National Center for the Analysis of Violent Crime.

Ferguson SK, Dacey MC (1997). Anxiety, depression, and dissociation in women health care providers reporting a history of childhood psychological abus., Child Abuse e Neglect 21(10):941-952.

Fite PJ, Raine A, Stouthamer-Loeber M, Loeber R, Pardini DA (2010). Reactive and proactive aggression in adolescent males: Examining differential outcomes 10 years later in early adulthood. Criminal Justice and Behavior 37:141-157.

Fox JA, Levin J (1994). Overkill: Mass murder and serial killing exposed. New York: Plenum.

Freud S (1924). The dissolution of the Oedipus complex. In J. Strachey (a cura di), The standard edition of the complete psychological works of Sigmund Freud. London: Hogarth Press, pp. 173-182.

Freud S (1960). The Ego and the Id, The Standard Edition. New York, Norton.

Garbarino J (2011). Not all bad treatment is psychological maltreatment. Child Abuse \& Neglect, 35:797-801.

Gibbons J, Conroy S, Bell C (1995). Operating The Child Protection System. London: HMSO Publication.

Gilbert P, Procter,S (2006). Compassionate mind training for people with high shame and self-criticism: Overview and pilot study of a group therapy approach. Clinical Psychology \& Psychotherapy 13:353-379.

Giusti E (1995). Autostima - psicologia della sicurezza in sé. Sovera: Roma.

Glaser D (2000). Child abuse and neglect and the brain - a review. Journal of Child Psychology and Psychiatry 41(1):97-116.

Glenn AL, Raine A (2009). Psychopathy and instrumental aggression: Evolutionary, neurobiological, and legal perspectives. International Journal of Law and Psychiatry 32:253-258.

Hale RL (1993). The application of learning theory to serial murder, or "You too can learn to be a serial killer". American Journal of Criminal Justice, 17(2):37-45

Hemple AG, Meloy JR, Richards TC (1999). Offender and offense characteristics of a nonrandom sample of mass murderers. Journal of the American Academy of Psychiatry and the Law 27(2):213-225.

Henry WP, Schacht TE, Strupp HH (1990). Patient and therapist introject, interpersonal process, and differential psychotherapy outcome. Journal of Consulting and Clinical Psychology 58:768-774.

Herman JL (1997). Trauma and recovery. New York: Basic Books.

Hickey EW (1997). Serial murderers and their victims, 2nd ed. Belmont: Wadsworth.

Higgins DJ, McCabe MP (2000). Relationships between different types of maltreatment during childhood and adjustment in adulthood. Child Maltreatment 5:261-272.

Hildyard KL, Wolfe DA.(2002). Child neglect: Developmental issues and outcomes. Child Abuse \& Neglect 26:679-695

Holmes RM, Holmes ST (1998). Selected problems in serial murder investigations. In: Contemporary perspectives on serial murder. Thousand Oaks: Sage, pp. 227-234.

Holmes ST, Tewksbury R, Holmes RM (1999). Fractured identity syndrome: A new theory of serial murder. Journal of Contemporary Criminal Justice 15:262-272.

Horwitz AV, Widom CS, McLaughlin J, White HR (2001). The impact of childhood abuse and neglect on adult mental health: a prospective study. Journal of Health \& Social Behavior 42(2):184-201.

Johnson EA, O'Brien KA (2013), Self-compassion soothes the savage ego-threat system: Effects on negative affect, shame, rumination, and depressive symptoms. Journal of Social and Clinical Psychology 32:939-963.

Karlin NJ (1995). Munchausen syndrome by proxy. Brattleboro Retreat Psychiatry Review 4(1):199.

Knoll JL (2010a). The "pseudocommando" mass murderer: Part I, The psychology of revenge and obliteration. Journal of the American Academy of Psychiatry and the Law 38:87-94.

Knoll JL (2010b). The "pseudocommando" mass murderer: Part II, The language of revenge. Journal of the American Academy of Psychiatry and the Law 38:263-272

Krahé B (2015). L'aggressività. In: Introduzione alla psicologia sociale. Bologna: Il Mulino.

Luberti R, Pedrocco Biancardi MT (2005). La violenza assistita intrafamiliare. Percorsi di aiuto per i bambini che vivono in famiglie violente. Milano: Franco Angeli.

MacCulloch MJ (1985). Mental disorder, multiple attacks and mass murder. In: P. Pichot, P. Berner, R. Wolf, K. Thau (a cura di) Psychiatry. Boston: Springer, p. 453-458.

Macdonald JM (1963). The threat to kill. American Journal of Psychiatry 120(2):125-130.

Main M, Solomon J (1986). Discovery of a disorganized/disoriented attachment pattern. n T. B. Brazelton, M.W. Yogman (a cura di), Affective development in infancy. New York: Ablex Publishing, pp. 95-124.

Main M, Solomon J (1990). Procedure for identifying infants as disorganised/disoriented during the Ainsworth Strange Situation. In: M. Greemberg, D. Cicchetti, M. Cummings (a cura di), Attachment during preschool years. Chicago: University Chicago Press, p. 121-160.

Marono AJ, Reid S, Yaksic E, Keatley DA (2020). A behaviour sequence analysis of serial killers' lives: From childhood abuse to methods of murder. Psychiatr Psychol Law 27(1):126-137.

Marsee MA, Frick PJ (2007). Exploring the cognitive and emotional correlates to proactive and reactive aggression in a sample of detained girls. Journal of Abnormal Child Psychology 35:969-981.

Mastronardi MV (2016). La comunicazione in famiglia. Prevenire il disagio e la devianza. Roma: Armando Editore.

Mastronardi MV, Palermo GB (1995). Meccanismi psicologici del serial killer e classificazioni. Video Documentario Scientifico 
presentato presso la Corte di Cassazione. Convegno Mostri o Serial Killer, 1-2 dicembre 1995.

Mastronardi MV, Palermo GB (2000). Psicopatologia di un omicida di massa; "Il singolare caso di Dion Terres". Rassegna di psicoterapie, Ipnosi, Medicina psicosomatica, Psicopatologia forense Vol. 5 n. 3

Morganett SR (2005). Yaşam Becerileri [Life skills]. Traduzione di: Sonay Gürçay, Alim Kaya, Mesut Saçkes). Ankara: Pegem Publishing.

Neff KD (2003). The development and validation of a scale to measure self-compassion. Self and Identity 2:223-250.

Newton M (1990). Hunting humans: An encyclopedia of modern serial killers. Atlanta: Breakout Productions.

Novello L (2018). Le violenze sui minori: definizioni e caratteristiche. In: P. Romito, N. Folla, M. Melato (a cura di), La violenza su donne e minori: Una guida per chi lavora su campo. Roma, Carrocci Editore.

Palermo GB, Mastronardi MV (2005). Il profilo criminologico: dalla scena del crimine ai profili socio-psicologici. Milano: Giuffrè Editore.

Pişkin M (2004). Özsaygıyı geliştirme eğitimi [Self-esteem development training]. Ankara: Nisan Publishing.

Radke-Yarrow M, Sherman T (1990). Hard growing: children who survive. In: J.E. Rolf, A.S. Masten, D. Cicchetti, K.H. Nuechterlein, S. Weintraub (a cura di), Risk and protective factors in the development of psychopathology. Cambridge: Cambridge University Press, pp. 97-119.

Raine A, Dodge,K, Loeber R, et al. (2006). The reactive-proactive aggression questionnaire: Differential correlates of reactive and proactive aggression in adolescent boys. Aggressive Behavior 32:159-171.

Rappaport N, Thomas C (2004). Recent research findings on aggressive and violent behavior in youth: Implications for clinical assessment and intervention. Journal of Adolescent Health 35:260-277.

Reif A, Rosler M, Freitag CM, et al. (2007). Nature and nurture predispose to violent behaviours: serotonergic genes and adverse childhood environment. Neuropsychopharmacology $32: 2375-23836$.

Rosenberg M (1965). Society and the adolescent self-image. Princeton: Princeton University Press.

Ross ND, Kaminski PL, Herrington R (2019). From childhood emotional maltreatment to depressive symptoms in adulthood: The roles of self-compassion and shame. Child Abuse \& Neglect 92:32-42.

Rowan AB, Foy DW (1993). Post-traumatic stress disorder in child sexual abuse survivors: A literature review. Journal of Traumatic Stress 6:3-20.

Save The Children Italia (2018). Le diverse forme dell'abuso all'infanzia: una pocket guide delle definizioni, gli obblighi e le responsabilità. https://www.savethechildren.it/sites/default/ files/files $/ 1 \% 20 \% 20$ CSP $\% 20$ Pocket $\% 20$ guide $\% 20$ su $\% 20$ abus o\% 20 con $\% 20$ casi $\% 20$ csp.pdf

Shackman JE, Pollak SD (2014). Impact of physical maltreatment on the regulation of negative affect and aggression. Development and Psychopathology 26:1021-1033.

Sullivan HS (1953). The interpersonal theory of psychiatry. New York: Norton.

Toppo G (2009). 10 years later, the real story behind Columbine. USA Today. http://usatoday30.usatoday.com/news/nation/ 2009-04-13-columbine-myths N.htm

Valente SM (2005). Sexual abuse of boys. Journal of Child and Adolescent Psychiatric Nursing 18:10-16

Vandello JA, Bosson JK, Cohen D (2008). Precarious manhood. Journal of Personality and Social Psychology 95:1325-1339.

Varese F, Smeets F, Drukker M, et al. (2012). Childhood adversities increase the risk of psychosis: A meta-analysis of patientcontrol, prospective- and cross-sectional cohort studies. Schizophrenia Bulletin 38:661-671.

Wyatt GE, Newcomb MD (1990). Internal and external mediators of women's sexual abuse in childhood. Journal of Consulting and Clinical Psychology 58:758.
Correspondence: Giulia Pitzanti.

E-mail: giulia.pitzanti@icloud.com

Key words: child abuse; aggressivity; mass murderer.

Parole chiave: maltrattamento infantile; aggressività; serial killer.

Palabras clave: abuso infantil; agresividad; serial killer.

Received for publication: 13 July 2021.

Accepted for publication: 21 August 2021

This article is distributed under the terms of the Creative Commons Attribution Noncommercial License (by-nc 4.0) which permits any noncommercial use, distribution, and reproduction in any medium, provided the original author(s) and source are credited.

${ }^{\circ}$ Copyright: the Author(s), 2021

Licensee PAGEPress, Italy

Rivista di Psicopatologia Forense, Medicina Legale, Criminologia $2021 ; 26: 631$

doi:10.4081/psyco.2021.631 